\title{
On the Interior Regularity of Weak Solutions to Nonlinear Elliptic Systems of Second Order
}

\author{
J. DAN ĚČKK
}

Es wird die $C^{1, \alpha}$.Regularität der schwachen Lösung (mit dem. Gradienten im BMO-Raum) eines nichtlinearen elliptischen Systems partieller Differentialgleichungen- zweiter Ordnung untersucht. Das Problem ist unter der Vóraussetzung lösbar, da B das System die verallgemeinerte Liouvillesche Bedingung im BMO-Raum statt wie gewöhnlich im $L^{\infty}$-Raum erfüllt. Zum Schluß wird gezeigt, daß die Liouvillesche Bedingung im Fall des $\mathbf{R}^{2}$ gilt.

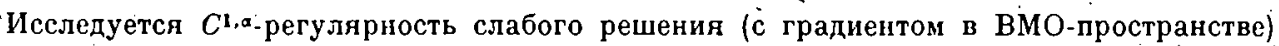
иелинейной эллиптической системы дифференциальных уравнений второго порядка. Проблема положительно разрешима в предположении, что система удовлетворяет обобщениому условию Лиувилля в ВМО-пространстве вместо как обычно в $L^{\infty}$-пространстве. В конце доказано, что условие Лиувилля выпольнено в случае $\mathbf{R}^{2}$.

The interior $C^{\text {l.a }}$-regularity for a weak solution (with gradient in the BMO-space) of a nonlinear second order elliptic system is investigated. The positive answer is obtained on the assumption that the elliptic system satisfy the generalized Liouville condition considered in the BMO-space instead of the usually used $L^{\infty}$-space. Finally it is proved that the Liouville condition holds in the case of $\mathbf{R}^{2}$.

\section{Introduction}

In this paper, which is a modified version of the thesis [4], we prove regularity for a weak-solution (with gradient in the BMO-space) of the-following nonlinear elliptic system $(i=1, \ldots, N)$ :

$$
-D_{\mathrm{a}}^{-} a_{i}{ }^{\alpha}(x, u, D u)+a_{i}(x, u, D u)=-D_{a} f_{i}{ }^{a}(x)+f_{i}(x),
$$

- where $x$ belongs to a bounded open set $\Omega$ of $\mathbf{R}^{n}, n \geqq 3, u: \Omega \rightarrow \mathbf{R}^{N}, N>1, u(x)$ $=\left(u^{1}(x), \ldots ., u^{N}(x)\right)$ is a vector-valued function, $D u=\left(D_{1} u, \ldots, D_{n} u\right), D_{a}=\partial / \partial x_{a}$; we will use the summation convention over repeated indices:

In $[6-9,12]$ the so-called Liouville condition $(\mathrm{L})$ is formulated in terms of the space $L^{\infty}$. On the other hand, the proof of $L^{\infty}$-boundedness of the gradient of a weak solution for the system $(0.1)$ has not yet been achieved in reasonably wide extent and the possibility of this proof is questionable.

The following definition is a generalized form of the Liouville property from $[7,8]$ and reads as follows.

Definition 0.1 : The system (0.1) satisfies the Liouville property (L) if for every $x^{0} \in \Omega$ and every $u \in \mathbf{R}^{N}$ the only solutions $v$ in $\mathbf{R}^{n}$ to

$$
-D_{a} a_{i}{ }^{\alpha}\left(x^{0}, u, D v(x)\right)=0, \quad(i=1, \ldots, N)
$$

with $D v \in \mathrm{BMO}\left(\mathbf{R}^{n}\right)$ are polynomials of at most first degree. 
The main result of this paper is the fact that if system $(0.1)$ has property $(L)$, then $D u$ is locally Hölder continuous in $\Omega$. To this effect it represents a generalization of $[7,8]$. Because it is easier to verify that the gradient of the solution is an element of the BMO-space $\left(L^{\infty} \subsetneq \mathrm{BMO}\right)$, the generalization reached in this paper has a fundamental meaning: The approach stated in this paper has been used in [15], which deals with quasilinear parabolic systems.

\section{Notations and definitions}

In the sequel $\Omega$ will be-a bounded open set of $\mathbf{R}^{n}$ with Lipschitz boundary $\partial \Omega$. The meaning of $\Omega_{0} \subset \subset \Omega$ is that the closure of $\Omega_{0}$ is contained in $\Omega$, i.e. $\bar{\Omega}_{0} \subset \Omega$. For the sake of simplification we denote by $|\cdot|$ and $(\cdot, \cdot)$-the norm and scalar product in $\mathbf{R}^{n}$.as well as in $\mathbf{R}^{N}$ and $\mathbf{R}^{n N}$. If $x \in \mathbf{R}^{n}$ and $r$ is a positive real number, we set $B(x, r)$ $=\left\{y \in \mathbf{R}^{n}:|y-x|<r\right\}, \Omega(x, r)=\Omega \cap B(x, r)$ and $Q(x, r)$ will be the cube in $\mathbf{R}^{n}$ with the center in the point $x$ and length of the side $r$.

By $\mathscr{P}_{k}, k \geqq 0$ integer, we denote the set of all vector-valued polynomials $P=\left(P^{1}\right.$, $\left.\ldots, P^{N}\right)$ with real coefficients defined on $\mathbf{R}^{n}$ such that the degree of $P^{i}$ is less than $k$ for each $i=1, \ldots, N$.

Beside the usually used Hölder and Soboler spaces (for detailed information see, e.g., $[3,6,12])$ we will use the following ones.

Definition 1.1 (Campanato-Morrey spaces): Let $\lambda \in[0, n], p \in[1, \infty)$. The space ${ }^{-}$ $L^{p, \lambda}(\Omega)$ is the subspace of such functions $f \in L^{p}(\Omega)$ for which

$$
\|f\|_{L p, \lambda(\Omega)}=\left\{\sup _{x \in \bar{\Omega}, r>0} r^{-\lambda} \int_{\Omega(x, r)}|f(y)|^{p} d y\right\}^{1 / p}<\infty .
$$

Let $k$ be a non-negative integer and $\lambda \in[0, n+(k+1) p]$ : The space $\mathscr{L}_{k}{ }^{p, \lambda}(\Omega)$ is the subspace of such functions $f \in L^{p}(\Omega)$. for which

where

$$
\|f\|_{Y_{k} p, \lambda(\Omega)}=\|f\|_{L p(\Omega)}+[f]_{Y_{k} p, \lambda(\Omega)}<\infty,
$$

$$
[f]_{\mathscr{L}_{k} p, \lambda\left(\Omega_{)}\right)}=\left\{\sup _{x \in \bar{\Omega}, r>0}\left[r^{-2} \inf _{P_{\in} \mathcal{S}_{\dot{k}}} \int_{\Omega_{(x, r)}}|f(y)-P(y)|^{p} d y\right]\right\}^{1 / p}
$$

With the norms (1.1) and (1.2), $L^{p, \lambda}(\Omega)$ and $\mathscr{L}_{k}^{p, \lambda}(\Omega)$ are Banach spaces. We will work mainly with the spaces $L^{2, \lambda}, \mathscr{L}_{0}^{2, \lambda^{\prime}}$ and $\mathscr{L}_{1}^{2, \lambda}$; instead of $\mathscr{L}_{0}^{2, \lambda}$ we will usually write $\mathscr{L}^{2, \alpha}$.

In our considerations we make use of the fact that for each function $u \in \mathscr{L}_{k}^{2, \lambda}(\Omega)$ each $x^{0} \in Q, 0<\ddot{r} \leqq \operatorname{diam} \Omega$, there exists one and only one polynomial $P \in \mathscr{P}_{k}$, $P(x)=P\left(x, x^{0}, r, u\right)$ such that

$$
\inf _{P \in \mathscr{P}_{k}} \int_{\left(x^{0}, r\right)}|u(x)-P(x)|^{2} d x=\int_{\Omega\left(x^{0}, r\right)}\left|u(x)-P\left(x, x^{0}, r, u\right)\right|^{2} d x
$$

For $k=1$ we will write this polynomial $\dot{P}$ in the form

$$
\begin{aligned}
\bar{P}\left(x, x^{0}, r, u\right) & =b^{0}\left(x^{0}, r, u\right)+\sum_{\alpha=1}^{n} b^{\alpha}\left(x^{0}, r, u\right)\left(x_{\alpha}-x_{\alpha}{ }^{0}\right) \\
& =b^{0}\left(x^{0}, r, u\right)+.\left(b\left(x^{0}, r, u\right),\left(x-x^{0}\right)\right)
\end{aligned}
$$

and for $k=0$ it equals the constant

$$
\therefore \quad \therefore u_{x^{0}, r}=\underset{B\left(x^{0}, r\right)}{f} u(y) d y=\left(\operatorname{meas} B\left(x^{0}, r\right)\right)^{-1} \int_{B\left(x^{0}, r\right)} u(y) d y
$$


where meas $B\left(x^{0}, r\right)$ means the $n$-dimensional Lebesgue measure. Denote further $U\left(x^{0}, r\right)=f_{B\left(x^{0}, r\right)}\left|u(y)-u_{x, r}\right|^{2} d y$, and define, $\operatorname{BMO}\left(\mathbf{R}^{n}\right)$ as the set of all measurable functions $u$ on $\mathbf{R}^{n}$ for which the set $u=\left\{U(x, r): x \in \mathbf{R}^{n}, r>0\right\}$ is bounded, setting $\|u\|_{\mathbf{B M I O}\left(\mathbf{R}^{n}\right)}=\sup u$.

At last, let $H^{1 .(\lambda)}(\Omega), \lambda \in[0, n]$ be the Banach space of all functions $u \in H^{1}(\Omega)$, $D_{a} u \in \mathscr{L}^{2, \lambda}(\Omega)$ with norm.

$$
\|u\|_{H 1,(\lambda)(\Omega)}=\|u\|_{L^{\prime}(\Omega)}+\sum_{\alpha=1}^{n}\left\|D_{\alpha} u\right\|_{Y^{2, \lambda(\Omega)}} \cdot
$$

Proposition 1.1: We have the following important properties of the spaces defined above:

(a) $L^{2, \lambda}(\Omega)=\mathscr{L}^{2, \lambda}(\Omega) ; \lambda \in[0, n)$,

(b) $\mathscr{L}^{2, \lambda}(\Omega)^{-}=\mathscr{L}_{1}^{2, \lambda}(\Omega), \lambda \in[0, n+2)$,

(c) $\mathscr{L}^{2, n}(\Omega) \subset L^{2, \lambda_{1}}(\Omega) \subset L^{2, \lambda_{2}}(\Omega), 0 \leqq \lambda_{2}<\lambda_{1}<n$;

(d) $L^{2, n}(\Omega)=L^{\infty}(\Omega) \subsetneq \mathscr{L}^{2, n}(\Omega)$,

(e) $\mathscr{L}^{p . n}(\Omega)=\mathscr{L}^{s . n}(\Omega)=\operatorname{BMO}(\Omega)$ for all $\dot{p}, s \in[1, \infty), \Omega$ being a cube,

(f). $H^{1,(n)}(\Omega) \subset C^{0 . \gamma}\left(\Omega_{0}\right)$ for each $\Omega_{0} \subset \subset \Omega, \gamma \in(0,1)$ and .

$\|\cdot\|_{c 0 . \gamma\left(\Omega_{0}\right)} \leqq c\left(n, \gamma, \operatorname{diam} \Omega, \operatorname{dist}\left(\Omega_{0}, \partial \Omega\right)\right)\|\cdot\|_{H 1,(n)(\Omega)}$.

For the proofs and more detailed information about the Campanato-Morrey spaces see, e.g., $[1-3,6,12]$. In the sequel we will denote all important constants by the symbol $\mathscr{C}$ and other' ones by $c$.

A function $u \in H^{1}(\Omega)$ is called weak solution of $(0.1)$ in $\Omega$ if

$$
\begin{aligned}
& \int_{\Omega} a_{i}{ }^{a}(x, u, D u) D_{a} \varphi^{i}(x) d x+\int_{\Omega} a_{i}(x, u, D u) \varphi^{i}(x) d x \\
& =\int_{\Omega} f_{i}{ }^{a}(x) D_{\alpha} \varphi^{i}(x) d x+\int_{\Omega} f_{i}(x) \varphi^{i}(x) d x \quad \text { for all } \varphi \in C_{0}^{\infty}(\Omega),
\end{aligned}
$$

where $a_{i}^{\alpha}, a_{i}^{i}, f_{i}^{\alpha}, f_{i}$ are functions fulfilling for each $\cdot(x, u, p) \in \Omega \times \mathbf{R}^{N} \times \mathbf{R}^{n \dot{N}}$ with $|u| \leqq L$ the following conditions:

$$
\begin{aligned}
& a_{i}{ }^{\alpha}, a_{i} \in \dot{C}^{1}\left(\Omega \times \mathbf{R}^{N} \times \mathbf{R}^{n N}\right) \\
& \left|a_{i}^{\alpha}(x, u, p)\right|,\left|a_{i}(x, u, p)\right| \leqq \mathscr{C}_{1}(L)(1+|p|) \\
& \left|\partial a_{i}{ }^{a}(x, u, p) / \partial p_{j}{ }^{\beta}\right|,\left|\partial a_{i}(x, u, p) / \partial p_{j}{ }^{\beta}\right| \leqq \mathscr{C}_{1}(L) \\
& \left.\begin{array}{l}
\left|\partial a_{i}{ }^{\alpha}(x, u, p) / \partial u_{k}\right|,\left|\partial a_{i}{ }^{\alpha}(x, u, p) / \partial x_{s}\right| \\
\left|\partial a_{i}(x, u, p) / \partial u_{k}\right|,\left|\partial a_{i}(x, u, p) / \partial x_{s}\right|
\end{array}\right\} \leqq \mathscr{C}_{1}(L)(1+|p|), \\
& \partial a_{i}{ }^{\alpha}(\dot{x}, u, p) / \partial p_{j}{ }^{\beta} \text { is uniformly continuous on } \Omega \times \mathbf{R}^{N} \times \mathbf{R}^{n N} \text {, } \\
& \partial a_{i}{ }^{\alpha}(x, u, p) / \partial p_{p}{ }^{\beta} \rightarrow d_{i j}^{\alpha \beta}(x, u) \text { as }|p| \rightarrow \infty, \quad \text { for all }(x ; u) \in \Omega \times \mathbf{R}^{N} \\
& f_{i}{ }^{a} \in H^{1, q}(\Omega), \quad f_{i} \in H^{1, q / 2}(\Omega) ; \quad \bar{q}>n, \\
& \sum\left\|f_{i}{ }^{\circ}\right\|_{H 1, q(\Omega)}+\sum\left\|f_{i}\right\|_{H 1, q / 2(\Omega)} \leqq \mathscr{C}_{2} \text {, } \\
& \dot{\partial} a_{i}{ }^{\alpha}(x, u, p) / \partial p_{j}^{\beta} \eta_{a}{ }^{i} \eta_{\beta}{ }^{j} \geqq v(L)|\eta|^{2} \\
& \text { for all } \eta \in \mathbf{R}^{n N},(x, u, p) \in \Omega \times \mathbf{R}^{N} \times \mathbf{R}^{n N} \text {. }
\end{aligned}
$$

It is known that $u \dot{\epsilon} H_{\text {loc }}^{2}(\Omega)$ if the function $u$ fulfiles the conditions stated above (see, e.g., [3]). 


\section{The results}

Principal result of this paper is the following theorem.

Theorem 2:1: Let $u \in H^{1,(n)}(\Omega)$ be a weak solution of the system (0.1) and suppose that the conditions (1.5)-(1.13) hold: If the system (0.1) has the Liouville property (L), then $u \in C_{\mathrm{loc}}^{\mathrm{l}, 1-n / q}(\dot{\Omega)}$.

There arise two natural questions:

1. Do there exist systems of the form $(0.1)$ with weak solutions in the space $H^{1,(n)}(\Omega)$ ?

2. Under which assumptions has the system of the form $(0.1)$ the Liouville property (L)?

A partial answer on the first question is given in [5]. The problem of the $H^{1 .(1)}$ regularity of weak solutions is studied in detail in [3]. The second question is positively answered in the case of $n=2$ and $N>1$ by the following

Proposition 2.2: Let the system (0.1) satisfy conditions $(1.5)-(1.8),(1.11)-(1.13)$ and let $n=2$. Then it has property (L).

In the case $n \geqq 3, N>1$ some conditions under which linear elliptic systems with . $L^{\infty}$-coefficients, quasilinear or nonlinear systems, respectively; have property (L) are shown in [11], [13] and [10], respectively. From [14] it follows that there are nonlinear elliptic systems without property (L).

\section{Leīmmas}

The following two lemmas concern the estimate of the coefficients of the polynomials from (1.5).

Lemma 3.1 [1: pp. 140-144]: Let $P \in \mathcal{P}_{k}, s \in[1, \infty)$ and $E$ be a measurable subset of the ball $B\left(x^{0}, r\right) \subset \mathbf{R}^{n}$ satisfying the condition meas $E \geqq A r^{n}, A$ a positive constant. Then there is a constant $c=c(n, k, s, A)$ such that for each multiindex $\alpha$ we have

$$
\left|\left[D_{a} P(x)\right]_{x=x_{0}}\right|^{s} \leqq\left(c / r^{n+|\alpha| s}\right) \int_{E}|P(x)|^{\varepsilon} d x
$$

Lemma 3.2 [1: pp. 146]: Let $u \in \mathscr{L}_{1}^{2, n+2}(\Omega)$. Then there exists a constant $c=c(n)$ such that for every $x \in \Omega$ and for all' $r, r_{0}, 0<r \leqq r_{0} \leqq \operatorname{diam} \Omega$, we have

$$
\begin{aligned}
& \left|b^{0}\left(x, r_{0}\right)-b^{0}(x, r)\right| \leqq c r_{0}[u]_{\mathscr{X}_{1} 2, n+2(\Omega)}, \\
& \left|b^{\alpha}\left(x, r_{0}\right)-b^{\alpha}(x, r)\right| \leqq c\left(1+\ln \left(r_{0} / r\right)\right)[u]_{\mathscr{Y}_{2} 2, n+2(\Omega)}
\end{aligned}
$$

for all $\alpha=1, \ldots, n$, where $b^{0}, b^{a}$ are defined in (1.3).

Another important result needed for the proof of Theorem 2.1 is the following

Proposition 3.3 [2: pp. 373] : Let- $\Omega$ be convex. Then there is a constant $c=c(n$, $\operatorname{diam} \Omega$, meas $\Omega$ ) such that for each $\lambda \in[0, n+2]$ we have

$$
\begin{aligned}
& H^{1,(\lambda)}(\Omega) \subset \mathscr{L}_{1}^{2, \lambda+2}(\Omega), \\
& \|u\|_{\dot{Y}_{1}, \lambda+2(\Omega)} \leqq c\|u\|_{H 1 .(\lambda)(\dot{\Omega})} \quad \text { for all } u \in H^{1,(\lambda)}(\Omega) .
\end{aligned}
$$


Now we presént a fundamental result concerning the partial regularity of weak solutions to the quasilinear elliptic systems of the type

$$
D_{\mathrm{a}}\left[A_{i j}^{\alpha \beta}(x, u) D_{\beta} u^{j}\right]+A_{i j}^{\beta}(x, u) D_{\beta} u^{j}=-D_{a} g_{i}^{\alpha}+g_{i} .
$$

Assume that the coefficients $A_{i j}^{\alpha \beta}$ are-uniformly continuous, $A_{i j}^{\beta}$ are continuous in $\Omega \times \mathbf{R}^{N}, g_{i}^{a} \in L^{q}(\Omega), g_{i} \in L^{q / 2}(\Omega), q>n$ and that $(c, \mu>0$ constants)

$$
\begin{aligned}
& \sum_{i, j, \alpha, \beta}\left|A_{i j}^{\alpha \beta}\right|+\sum_{i, j, \beta}\left|A_{i j}^{\beta}\right|+\sum_{i, \alpha}\left\|g_{i}^{\alpha}\right\|_{L^{\alpha}}+\sum_{i}\left\|g_{i}\right\|_{L Q / 2} \leqq c, \\
& A_{i j}^{\alpha \beta}(x, u) \xi_{a}^{i} \xi_{\beta^{j}} \geqq \mu|\xi|^{2} \quad \text { for all }(x, u) \in \Omega \times \mathbf{R}^{N}, \quad \xi \in \mathbf{R}^{n N} .
\end{aligned}
$$

Consider the solutions to the system (3.1) belonging to the space $H^{1} \cap \mathscr{L}^{2, n}(\dot{Q})$.

Proposition 3.4 [12: pp. 147-149]: Let $u$ be a weak solution of the system (3.1). Suppose that $U(x, r) \rightarrow 0$ as $r \rightarrow 0+$ uniformly in each compact set $K \subset \Omega$. Then $u \in C_{10 \mathrm{c}}^{0, \alpha}(\Omega)$ with $\alpha=1-n / q$ and the a-priori estimate $\|u\|_{c 0, \alpha(K)} \leqq c_{1}(\mu, c, K$, dist $(K$, $\partial \Omega))$ hólds.

\section{Proof of the results}

Iet $\Omega_{0} \subset \subset \Omega, x^{0} \in \Omega_{0}$ be fixed; $R_{0}=\min \left\{1\right.$, dist $\left.\left(\Omega_{0} ; \partial \Omega\right)\right\}$. For $R \in\left(0, R_{0}\right)$ and $-u \in H^{1,(n)}(\Omega)$ ( $u$ is a weak solution of the system $\left.(0.1)\right)$ we define

$$
\begin{aligned}
& y=y(x)=\left(x-x^{0}\right) / R, \\
& u_{R}(y)=\left(u\left(x^{0}+R y\right)-b^{0}\left(x^{0}, R\right)-R\left(b\left(x^{0}, R\right), y\right)\right) / R,
\end{aligned}
$$

where $b^{0}\left(x^{0}, R\right)=b^{0}\left(x^{0}, R, u\right) \in \mathbf{R}^{N}$ and $b\left(x^{0}, R\right)=b\left(x^{0}, R, u\right) \in \mathbf{R}^{n N}$ are the coefficients of the polynomial $P\left(x, x^{0}, R, u\right)$ from (1.3) since $u \in \cdot \mathscr{L}_{1}^{2 . n+2}\left(B\left(x^{0}, R\right)\right)$ for each $B\left(x^{0}, R\right) \subset \Omega$ due to Proposition 3.3. From (4.1) it can be seen that for each $a>0$ there exists $R(a) \in\left(0, R_{0}\right]$ such that for all $R \in(0,-R(a))$ we have $B(0,2 a \sqrt{n}) \sqsubset O_{R}$ $\left(O_{R}\right.$ is the image of $\Omega$ through the transformation (4.1)). From (4.2) it follows that there exists a constant $c>0$ such that for each $r>0, y^{0} \in \mathbf{R}^{n}$ and all $R \in\left(0, R\left(y^{0}\right)\right)$ $\left(R\left(y^{0}\right)=R_{0}\right.$ in the case $\left.y^{0}=0\right)$ we have

$$
\int_{B\left(y^{\circ}, r\right)}\left|D u_{R}(y)-\left(D u_{R}\right)_{y^{0}, r}\right|^{2} d y \leqq c[D u]_{Y 2, n(\Omega)} r^{n}
$$

and the equation (1.4) has the following form:

$$
\begin{aligned}
& \int_{O_{R}} a_{i}^{\alpha}\left(\dot{x}^{0}+R y, b^{0}\left(x^{0}, R\right)+R u_{R}(y)+R\left(b\left(x^{0}, R\right), y\right), b\left(x^{0}, R\right)+D u_{R}(y)\right) D_{a} \psi^{i}(y) d y \\
& +\int_{O_{n}} R a_{i}\left(x^{0}+R y, b^{0}\left(x^{0}, R\right)+R u_{R}(y)+R\left(b\left(x^{0}, R\right), y\right), b\left(x^{0}, \ddot{R}\right)+D u_{R}(y)\right) \psi^{i}(y) d y \\
& =\int_{O_{R}} f_{i}^{\alpha}\left(x^{0}+R y\right) D_{a} \psi^{i}(y) d y+\int_{O_{R}} R f_{i}\left(x^{0}+R y\right) \psi^{i}(y) d y \quad \text { for all } \psi \in C_{0}^{\infty}\left(O_{R}\right) \text {. }
\end{aligned}
$$

As previously'said, $u \in H_{\text {loc }}^{2}(\Omega)$ and with respect to (4.2) also $u_{R} \in H_{1 \text { oc }}^{2}\left(O_{R}\right)$. Then it follows that $v_{R}=D_{y} u_{R}$ satisfies the equation in variations

$$
\begin{aligned}
& \int_{o_{R}}\left(\partial a_{i}{ }^{\alpha} / \partial p_{j}^{\beta} D_{\beta} v_{R}{ }^{j}+R \partial a_{i}{ }^{\alpha} / \partial u^{k}\left(b_{k}{ }^{\gamma}+\dot{v}_{R}{ }^{k}\right)+R \partial a_{i}^{\alpha} / \partial x_{\gamma}\right) D_{a} \psi^{i} d y \\
& +\int_{o_{R}}\left(R \partial a_{i} / \partial p_{j}{ }^{\beta} D_{\beta} v_{R}{ }^{j}+R^{2} \partial a_{i} / \partial u^{k}\left(b_{k}{ }^{\gamma}+v_{R}{ }^{k}\right)+R^{2} \partial a_{i} / \partial x_{\gamma}\right) \psi^{i} d y \\
& -\int_{O_{R}}\left(R \partial f_{i} / \partial x_{\gamma} D_{\alpha} \psi^{i}+R^{2} \partial f_{i} / \partial x_{i}\right) \psi^{i} d y \quad \text { for all } \psi \in C_{0}{ }^{\infty}\left(O_{R}\right) .
\end{aligned}
$$


In what follows we are going to prove that for each $a>0$ the set $\mathcal{M}_{0}=\left\{u_{R}\right.$ : $0<R<R(a)\}$ is bounded in $H^{2}(B(0, a))$ by a constant depending only on $a$. For this. reason it is enough to prove the boundedness of sets $\mathscr{M}_{0}$ and $\mathscr{M}_{2}=\left\{D^{2} u_{R}: 0<R\right.$ $<R(a)\}$ in $L^{2}(B(0, a))$. The set $\mathscr{A}_{\mathrm{i}}=\left\{D u_{R}: 0<R<R(a)\right\}$ is then bounded according to the Gagliardo-Nirenberg Theorem (sce, e.g., [3: pp. 25]).

First, let us prove the boundedness of $\mathscr{M}_{2}$. For $a>0$ denote $B(a)=B(0, a)$. Further choose $\eta \in C_{0}^{\infty}(B(2 a))$ such that $0 \leqq \eta \leqq 1, \eta=\overline{1}$ on $B(a)$ and $|D \eta| \leqq c / a$. Substituting for $\psi$. in the equation (4.5) the function $\psi(y)=\eta^{2}\left[v_{R}(y)-\left(v_{R}\right)_{0,2} a\right.$, we have for each $\varepsilon>0$ from the assumptions $(1.7),(1.8),(1.11)-(1.13)$, Young's inequality, Proposition 1.1 and properties of the function $\eta$ that

$\nu(L) \int \eta^{2}\left|D v_{R}\right|^{2} d y$

$B(0,2 a)$

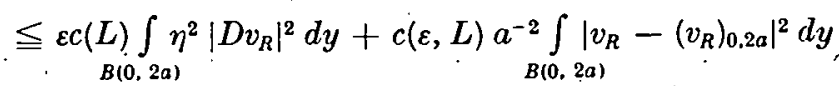

$$
+c(\varepsilon, L)\left\{R^{2}\left(1+\left|b\left(x^{0}, R\right)\right|_{B(0,2 a)}^{2}\right) \int_{B(0)}\left|\tilde{D} u_{R}\right|^{2} d y+\underset{B(0,2 a)}{R^{2}} \int_{\mid}\left|D u_{R}\right|^{4} d y\right.
$$

$$
\left.+R^{2}\left(1+\left|b\left(x^{0}, R\right)\right|^{2}+\left|b\left(x^{0} ; \underline{R}\right)\right|^{4}\right) a^{n}+R_{B(0,2 a)}^{2}|D \tilde{f}|^{2} d y+R_{B(0,2 a)}^{4}|D \tilde{f}|^{2} d y\right\} \text {, - }
$$

here $\tilde{f}=\left(f_{i}^{\alpha}\right), \tilde{f}=\left(f_{i}\right), L=L\left(\operatorname{dist}\left(\Omega_{0}, \partial \Omega\right), \operatorname{diam} \Omega ;\|u\|_{\left.H_{1,(n)(\Omega)}\right)}\right.$; in the case $q<4$ it is necessary to replace the last integral in (4.6) by $R^{q} \int|D f|^{q / 2} d y$. Choosing $\varepsilon>0$ in (4.6) small enough, we obtain

$$
\begin{aligned}
& \int_{B(0, a)}\left|D v_{R}\right|^{2} \dot{d} y \\
& \leqq c(L)\left\{a_{B(0,2 a)}^{-2}\left|v_{R}-\left\langle v_{R}\right)_{0,2 a}\right|^{2} d y+\dot{R^{2}}\left(1+\left|b\left(x^{0}, R\right)\right|_{B(0,2 a)}^{\dot{2}}\right) \cdot \int_{-2 a}\left|D u_{R}\right|^{2} \dot{d y}\right. \\
& +R^{2} \int\left|D u_{R}\right|^{4} d y+R^{2}\left(1+\cdot\left|b\left(x^{0}, R\right)\right|^{2}+\left|b\left(x^{0}, R\right)\right|^{4}\right) a^{a} \\
& \left.+{ }_{B(0,2 a)}^{B(0,2 a)} \int^{2}|D \tilde{f}|^{2} d y+R_{B(0,2 a)}^{4}|D \tilde{f}|^{2} d y\right\} \\
& =c(L)\{A+B+C+D+E+F\} \text {. }
\end{aligned}
$$

Estimate now the individual terms in brackets. Since $D i \in \mathscr{L}^{2 . n}(\Omega)$, we have

$$
A=a^{-2} R^{-n} \int_{B\left(x^{0}, 2 a R\right)}\left|\partial u / \partial x_{y}-\left(\partial u / \partial x_{y}\right)_{x^{0}, 2 a R}\right|^{2} d x \leqq c[D u]_{Y 2, n(\Omega)} a^{n-2}
$$

Further from Lemma 3.1, Lemma 3.2 and the fact that $D u \in L^{2, \lambda}(\Omega)$ for each $\lambda \in[0, n)$ - (according to Proposition 1.1/(c)) we obtain

$$
\begin{aligned}
B & =\left(1+\left|b\left(x^{0}, R\right)\right|^{2}\right) R^{-n+2} \int_{B\left(x^{0}, 2 a R\right)}\left|D u-b\left(x^{0}, R\right)\right|^{2} d x \\
& \leqq c\left[R^{\lambda+2-n}\left(1+\left|b\left(x^{0}, R\right)\right|^{2}\right) a^{\lambda}+R^{2}\left(\left|b\left(x^{0}, R\right)\right|^{2}+\left|b\left(x^{0}, R\right)\right|^{4}\right)\right] a^{n} \\
& \leqq c\left(\lambda, R_{0}\right)\left(1+\ln ^{4} R\right) R^{\lambda+2-n}\left(a^{\lambda}+a^{n}\right)\|u\|_{H 1,(n)(\Omega)} \\
& \leqq c\left(\lambda, \dot{R}_{0},\|u\|_{H 1,(n)(\Omega)}\right)\left(a^{\lambda}+a^{n}\right)
\end{aligned}
$$


where $\dot{\lambda} \in(n-2, n)$ is arbitrary. In estimating the term $C$ we use the fact that $D u \in L^{s, \mu}(Q)$ for each cube $Q \subset \Omega, s \in[1, \infty), \mu \in[0, n)$ (see Proposition $1.1 /(\mathrm{c})$ ) and we proceed analogously as in the estimation of term $B$ and obtain $C \leqq c\left(\lambda, R_{0}\right.$, $\left.\|u\|_{H(1, n)(\Omega)}\right) a^{\lambda}$, where $\lambda \in(n-2, n)$ is arbitrary. From Lemma 3.2 it follows that $D$ $\leqq c\left(R_{0}\right) a^{n}$ and from the assumptions (1.11), (1.12) we have $E \leqq c\left(R_{0}, \mathscr{C}_{2}\right) \dot{a}^{n(1-2 / q)}$,

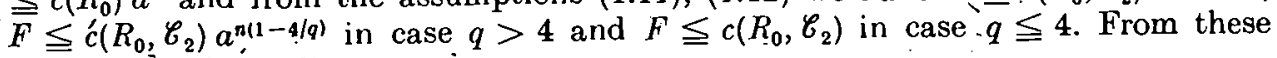
estimates it then follows

$$
\int_{B(0, a)}\left|D v_{R}\right|^{2} d y \leqq c\left(\mathscr{C}_{2}, R_{0}, \operatorname{diam} \Omega,\|u\|_{H 1,(n)(\Omega)}, a\right) \leqq c(a)
$$

for each $R \in(0, R(a))$. Hence $\int_{B(0, a)}\left|D^{2} u_{R}\right|^{2} d y \leqq c(a)$ for any $R \in(0, R(a))$ and the boundedness of the set $\mathscr{M}_{2}$ is proved.

Now we are going to prove the boundedness of $\mathcal{M}_{0}$. From Lemma 3.2, Propósition 3.3 and (4.1), (4.2) we have

$$
\begin{aligned}
\int_{B(0, a)}\left|u_{R}(y)\right|^{2} d y= & R^{-n-2} \int_{B\left(x^{0}, a R\right)}\left|u(x)-b^{0}\left(x^{0}, R\right)-\left(b\left(x^{0}, R\right),\left(x-x^{0}\right)\right)\right|^{2} d x \\
\leqq & 2 a^{n+2}(a R)^{-n-2} \int_{B\left(x^{0}, a R\right)^{\prime}}\left|u(x)-b^{0}\left(x^{0}, a R\right)-\left(b\left(x^{0}, a R\right),\left(x-x^{0}\right)\right)\right|^{2} d x \\
& +2 R^{-n-2} \int_{B\left(x^{0}, a R\right)} \mid b^{0}\left(x^{0}, a R\right)-b^{0}\left(x^{0}, R\right) \\
& +\left.\left(\left(b\left(x^{0}, a R\right)-b\left(x^{0}, R\right)\right),\left(x-x^{0}\right)\right)\right|^{2} d x \\
\leqq & c[u]_{Y, 2, n+2\left(B\left(x_{0}, a R\right)\right)}\left(1+\ln ^{2} a\right) \max \left\{a^{n}, a^{n+2}\right\} \leqq[D u]_{Y 2, n(\Omega)} c(a)
\end{aligned}
$$

Hence $\int_{B(0, a)}\left|u_{R}(y)\right|^{2} d y \leqq c(a)$ for any $R \in(0, R(a))$ and the boundedness of $\mathcal{M}_{0}$ in $H^{2}(B(0, a))$ is proved.

Compactness of the imbedding of $H^{2}(B(0, a))$ into $H^{1}(B(0, a))$ allows us to choose a sequence $\dot{R}_{k} \rightarrow 0$ such that $u_{R_{k}} \rightarrow z$ in $H^{1}(B(0, a))$. Using the diagonal process we get a subsequence (we use the same notation for it) such that

$$
\lim _{k \rightarrow \infty} u_{R_{k}}=\dot{z} \text { in } H_{\operatorname{loc}}^{1}\left(\mathbf{R}^{n}\right), \quad \lim _{k \rightarrow \infty} D u_{R_{k}}=D z \text {, a.e. in } \mathbf{R}^{n} \text {. }
$$

According to (4.3) we obtain that there exists a constant $c>0$ such that for each $y^{0} \in \mathbf{R}^{n}, r>0$ there holds

$$
\int_{B\left(y^{0}, r\right)}\left|D z(y)-(D z)_{\nu^{0}, r}\right|^{2} d y \leqq c[D u]_{Y 2, n(\Omega)}, r^{n} .
$$

Further we deduce from (4.4) the equation for the limit function $z$. For passing to the limit in equation (4.4) the behaviour of $\sup \left\{b\left(x^{0}, R_{k}\right), k=1,2, \ldots\right\}$ is important. Remember for the following considerations that $R b\left(x^{0}, R\right) \rightarrow 0, b^{0}\left(x^{0}, R\right) \rightarrow B^{0} \in \mathbf{R}^{N}$ as $R \rightarrow 0$ + exist due to Lemma 3.2 and from the definition of $u_{R}$ follows boundedness of the set $\left\{u_{R}: R>0\right\}$ by a constant independent of $R$.

(a) Let sup $\left\{\left|b\left(x^{0}, R_{k}\right)\right|: k=1,2, \ldots\right\}$ be a finite number. In this case there exists a subsequence (we use the same notation for it) $\left\{b\left(x^{0}, R_{k}\right)\right\}$ such that $b\left(x^{0}, R_{k}\right) \rightarrow B \in \mathbf{R}^{n N}$ as $k \rightarrow \infty$. According to (1.6), (1.12), (4.7) and the Vitali Convergence Theorem we can pass to the limit with $k \rightarrow \infty$ in the equation (4.4) (for the fixed function. $\psi$ ). We see that the second integral on the left-hand side and the integrals on the right-hand side in (4.4) tend to zero. Thus we obtain that $B+D z(y)$ is a weak solution of the system

$$
\int_{\mathbf{R}^{n}} a_{i}^{\alpha}\left(x^{0}, B^{0}, B+D_{z}\right) D_{\alpha} \psi^{i} d y=0 \quad \text { for all } \psi, \in H_{0}^{i}\left(\mathbf{R}^{n}\right)
$$


Now from the Liouville property of the system (1.4) it follows that $z$ is a polynomial of at most first degree.

(b) Let $\sup \left\{\left|b\left(x^{0}, R_{k}\right)\right|: k=1,2, \ldots\right\}$ be infinite. In this case we can suppose $\left|b\left(x^{0}, R_{k}\right)\right| \rightarrow \infty$ as $k \rightarrow \infty$. Denoting in the sequel $b_{k}=b\left(x^{0}, R_{k}\right), b_{k}^{0}=b^{0}\left(x^{0}, R_{k}\right)$, $u_{k}(y)=u_{R_{k}}(y), w_{k}(y) \doteq R_{k}\left(u_{R_{k}}(y)+\left(b\left(x^{0}, R_{k}\right), y\right)\right)$ we can rewrite equation (4.4) as follows:

$$
\begin{aligned}
& \int_{\mathbf{R}^{n}}\left[a_{i}^{\alpha}\left(x^{0}+R_{k} y, b_{k}{ }^{0}+w_{k}(y), b_{k}+D u_{k}(y)\right)-a_{i}^{\alpha}\left(x^{0}+R_{k} y, b_{k}{ }^{0}+w_{k}(y), b_{k}\right)\right. \\
& +a_{i}^{\alpha}\left(x^{0}+R_{k} y, b_{k}{ }^{0}+w_{k}(y), b_{k}\right)-a_{i}^{\alpha}\left(x^{0}+R_{k} y, b_{k}{ }^{0}, b_{k}\right) \\
& \left.+a_{i}^{\alpha}\left(x^{0}+R_{k} y, b_{k}{ }^{0}, b_{k}\right)-a_{i}^{\alpha}\left(x^{0}, b_{k}^{0}, b_{k}\right)\right] D_{a} \psi^{i}(y) d y \\
& +R_{k} \int_{\mathbf{R}^{n}} a_{i}\left(x^{0}+R_{k} y, b^{0}+w_{k}(y), b_{k}+D u_{k}(y)\right) \psi^{i}(y) d y \\
& =\int_{\mathbf{R}^{n}} f_{i}^{\alpha}\left(x^{0}+R_{k} y\right) D_{\alpha} \psi^{i}(y) d y+R_{k} \int_{\mathbf{R}^{n}} f_{i}\left(x^{0}+R_{k} y\right) \psi^{i}(y) d y \quad \text { for all } \psi \in C_{0}^{\infty}\left(\mathbf{R}^{n}\right) .
\end{aligned}
$$

Using the theorem on the mean value in the integrals from the previous system we can rewrite this system in the following form:

$$
\begin{aligned}
& \int_{\mathbf{R}^{n}} \int_{0}^{1} \partial a_{i}^{\alpha} / \partial p_{j}^{\beta}\left(x^{0}+R_{k} y, b_{k}{ }^{0}+w_{k}(y), b_{k}+t D u_{k}(y)\right) D_{\beta} u_{k}^{j}(y) D_{\alpha} \psi^{i}(y) d t d y, \\
& +R_{k} \int_{\mathbf{R}^{n}} \int_{0}^{1} \partial a_{i}^{\alpha} / \partial u^{8}\left(x^{0}+R_{k} y, b_{k}{ }^{0}+t i w_{k}(y), b_{k}\right) w_{k}^{s}(y) D_{\alpha} \psi^{i}(y) d t d y \\
& +R_{k} \int_{\mathbf{R}^{n}} \int_{0}^{1} \partial a_{i}^{\alpha} / \partial x_{\gamma}\left(x^{0}+t R_{k} y, b_{k}^{0}, b_{k}\right) y_{\gamma} D_{a} \psi^{i}(y) d t d y \\
& +R_{k} \int_{\mathbf{R}^{n}} a_{i}\left(x^{0}+R_{k} y, b_{k}^{0}+\dot{w_{k}}(y), b_{k}+D u_{k}(y)\right) \psi^{i}(y) d y \\
& =\int_{\mathbf{R}^{n}} f_{i}^{\alpha}\left(x^{0}+R_{k} y\right) D_{a} \psi^{i}(y) d y+R_{k} \int_{\mathbf{R}^{n}} f_{i}\left(x^{0}+R_{k} y\right) \psi^{i}(y) d y \quad \text { for all } \psi \in C_{0}{ }^{\infty}\left(\mathbf{R}^{n}\right)
\end{aligned}
$$

Taking into account (1.7), (1.9), (1.10), (1.12), (4.7) we can pass in the previous equation to the limit with $k \rightarrow \infty$ (for the fixed function $\psi$ ) and we have that the second, third and fourth integral in the left-hand side and the integrals on the right-hand side tend to zero. Due to $(1.10)$ and the assumption $\left|b\left(x^{0}, R_{k}\right)\right| \rightarrow \infty$ as $k \rightarrow \infty$, we obtain that the function $z$ satisfies the equation

$$
\int_{\mathbf{R}^{n}} d_{i j}^{\alpha \beta}\left(x^{0}, B^{0}\right) D_{\beta} z^{j} D_{\alpha} \psi^{i} d y=0 \quad \text { for all } \psi \in C_{0}{ }^{\infty}\left(\mathbf{R}^{n}\right)
$$

It is a linear elliptic system with the same constant of ellipticity and constant coefficients and by means of (4.8) we have that $D z \in \mathrm{BMO}\left(\mathbf{R}^{n}\right)$. In this case $z$ is a polynomial at most first degree again.

Returning to the $x$-coordinates, we prove that for each $x^{0} \in \Omega_{0}$ there exists a sequence $R_{k} \rightarrow 0$ such that

$$
\lim _{R_{x \rightarrow 0} \rightarrow 0} \underset{B\left(x^{0} . R_{k}\right)}{f}\left|D u(x)-(D u)_{x^{0}, R_{k}}\right|^{2} d x=0 .
$$


We have

$$
\begin{array}{r}
\underset{B\left(x^{0}, \dot{R}_{k}\right)}{f}\left|D u(x)-(D u)_{x^{0}, R_{k}}\right|^{2} d x=\int_{B(0,1)}\left|D u_{R_{k}}(y)-\left(D u_{R_{k}}\right)_{0,1}\right|^{2} d y \\
\qquad \underset{B(0,1)}{\leqq} \cdot \int_{R_{k}} \mid D u_{R^{2}} d y \quad \text { for all } t \in \mathbf{R}^{n_{N}} .
\end{array}
$$

Now we put $t=D z$ ( $D z$ is a constant) and, passing to the limit, we see that (4.9) holds.

Now let us consider the equation in variations for the system (1.4) in $\Omega_{0}$. If we denote by $v_{y}$ the derivative $D_{y} u$, we get as before that .

$$
\begin{aligned}
& \int_{\Omega_{0}}\left(\partial a_{i}{ }^{\alpha} / \partial p_{j}^{\beta} \cdot D_{\beta} v_{\gamma}{ }^{j}+\partial a_{i}^{\alpha} / \partial u^{k} v_{\gamma}{ }^{k}+\partial a_{i}^{\alpha} / \partial x_{y}\right) D_{\alpha} \varphi^{i} d x \\
& +\int_{\Omega_{0}}\left(\partial a_{i} / \partial p_{j}^{\beta} D_{\beta} v_{y}^{j}+\partial a_{i} / \partial u^{\hat{k}} v_{y}^{k}+\partial a_{i} / \partial x_{y}\right) \varphi^{i} d x \\
& =\int_{\Omega_{0}}\left(\partial f_{i}^{\alpha} / \partial x_{\gamma} D_{a} \varphi^{i}+\partial f_{i} / \partial x_{\gamma} \varphi^{i}\right) d x \text {. for all } \varphi \in C_{0}^{\infty}\left(\Omega_{0}\right), \quad \gamma=1, \ldots, n
\end{aligned}
$$

Set

$$
\begin{aligned}
& A_{i j}^{\alpha \beta}(x, v)=\partial a_{i}^{\alpha} / \partial p_{j}^{\beta}(x, u(x), v), \quad A_{i j}^{\beta}(x, v)=\partial a_{i} / \partial p_{\gamma}^{\beta}(x, u(x), v), \\
& g_{i}^{\alpha \gamma}(x)=-\partial a_{i}^{\alpha} / \partial u^{k}(x, u(x), D u(x)) v_{\gamma}{ }^{k}(x)-\partial a_{i}^{\alpha} / \partial x_{y}(x, u(x), D u(x))+\partial f_{i}^{\alpha} / \partial x_{\gamma}(x), \\
& g_{i}{ }^{\gamma}(x)=-\partial a_{i} / \partial u^{k}(x, u(x), D u(x)) v_{y}{ }^{k}(x)-\partial a_{i} / \partial x_{y}(x, u(x), D u(x))+\partial f_{i} / \partial x_{\gamma}(x) .
\end{aligned}
$$

From the assumption of the theorem it follows that $A_{i j}^{\alpha \beta}$ are uniformly continuous and bounded in $\Omega_{0} \times \mathbf{R}^{n N}, A_{i j}^{\beta}$ are continuous and bounded in $\Omega_{0} \times \mathbf{R}^{n N}, g_{i}^{\alpha \gamma} \in L^{q}\left(\Omega_{0}\right)$ and $g_{i}{ }^{\gamma} \in L^{q / 2}\left(\Omega_{0}\right)$. Then the system $(4: 10)$ can be rewritten as

$$
\begin{aligned}
& \int_{\Omega_{0}} \delta_{\theta_{\gamma}}\left[A_{i j}^{\alpha \beta}(x, v) D_{\beta} v_{\gamma}^{j} D_{\alpha} \varphi_{\theta}^{i}+A_{i j}^{\beta}(\dot{x}, v) D_{\beta} v_{\gamma}^{j} \varphi_{\theta}{ }^{i}\right] d x \\
& =\int_{\Omega_{0}}\left[g_{i}^{\alpha \theta}(x) D_{\alpha} \varphi_{\theta}{ }^{i}+g_{i}{ }^{\theta}(x) \varphi_{\theta}{ }^{i}\right] d x \text {, for all } \varphi \in C_{0}{ }^{\infty}\left(\Omega_{0}\right) .
\end{aligned}
$$

Thus $v$ is a solution of a quasilinear system of the type (3.1) for which partial regularity (Proposition 3.4) holds ((4.9) guarantees that the assumption of Proposition 3.4 is satisfied)

Proof of Proposition 2.2: Let $v \in H_{\text {loc }}^{1}\left(\mathbf{R}^{2}\right)$ with $D v \in \mathrm{BMO}\left(\mathbf{R}^{2}\right)$ be a weak solution in $\mathbf{R}^{2}$ of

$$
\int_{\mathbf{R}^{2}} a_{i}^{\alpha}\left(x^{0}, u, D v\right) D_{a} \varphi^{i}(x) d x=0 \quad \text { for all } \varphi \in C_{0}{ }^{\infty}\left(\mathbf{R}^{2}\right)
$$

The equation in variations is

$$
\text { 、 } \int_{\mathbf{R}^{1}} \partial a_{i}^{\alpha} / \partial p^{\beta}\left(x^{0}, u, D v\right) D_{\beta} \dot{v}_{y}^{j} D_{\alpha} \varphi^{i} d x=0 \quad \text { for all } \varphi \in C_{0}{ }^{\infty}\left(\mathbf{R}^{2}\right),
$$

where $v_{y}=D_{y} v$. Now we prove that $D v_{y} \in L^{2}\left(\mathbf{R}^{2}\right)$. Let $y^{0} \in \mathbf{R}^{2}, T>0$ be an arbitrary constant. Setting $\varphi^{i}=\eta^{2}\left(v_{\gamma}{ }^{i}-\left(v_{\gamma}{ }^{i}\right)_{\nu^{0} .2}\right), \eta \in C_{0}^{\infty}\left(B\left(y^{0}, 2 T\right)\right), 0 \leqq \eta \leqq 1, \eta=1$ in $B\left(y^{0}, T\right),|D \eta| \leqq c / T$ in equation (4.11), we get, $\int_{B\left(\nu^{\circ}, T\right)}\left|D v_{y}\right|^{2} d x \leqq c$ for $\gamma=1, \ldots, n$, where $c$ is independent of $y^{0}$ and $T$. It is known that a sequence $\left\{\varphi_{k}\right\} \subset C_{0}^{\infty}\left(\mathbf{R}^{2}\right)$ exists such that $D \varphi_{k} \rightarrow D v_{y}$ in $L^{2}\left(\mathbf{R}^{2}\right)$ and therefore from (4.11) we have

$$
\int_{\mathbf{R}^{\prime}} \partial a_{i}{ }^{\alpha} / \partial p_{i}{ }^{\beta}\left(x^{0}, u, D v\right) D_{\beta} v_{\gamma}{ }^{j} D_{a} v_{\gamma}{ }^{i} d x=0
$$

and together with the condition of ellipticity (1.13) gives the result 


\section{REFERENCES}

[1] Campanato, S.:- Proprieta di una famiglia di spazi funzionali. Ann. Scuola Nor. Sup. Pisa 18 (1964,) 137-160.

[2] Campanato, S.: Equazioni ellittiche del $\mathrm{II}^{0}$ ordine e spazi $\mathscr{L}(2, \lambda)$. Ann. Mat. Pura Appl. 69 (1965), $321-381$.

[3] Campaxato, S.: Sistemi ellittici in forma divergenza. Regolarita all'interno. Pisa: Scuola Normale Superiore 1980.

[4] Dañ Ěče, J.: Regularita slabých řešení nelineárních eliptických systémú. Thesis. Praha.: Mat.:Fyz. Fakulta Univ: Carol. 1984.

[5] DANĚČK, J.: Regularity for nonlinear elliptic šystems of second örder. Comment. Math. Univ. Carolinae 27. (1986), $755-764$.

[6] Graquista, M.: Multiple integrals in the calculus of variations and nonlinear elliptic systems: Princeton: University Press 1983.

[7] GIAqUiNTa, M., and J. NEČas: On the regularity of weak solutions tó nonlinear elliptic systems via Liouville's property. Comment. Math. Univ. Carolinae $20 \cdot(1979), 111-121$.

[8] Graquinta, M., and J. NeČas: On the rëgularitý of weak solutions to nonlinear elliptic systems of partial differential equations. J. Reine-Angew. Math. 316 (1980), 140-159.

[9] Graquinta, M.; NeČas, J., John, O., and J. Stará: On the regularity up to the boundary for second order nonlinear elliptic systems: Pac. J. Math. 99 (1982), 1-17.

[10] Кошелев, А. И.: О точных условиях гладкости решений әллиптических систем и теореме Лиувилля. Докл. Акал. Наук СССР 265 (1982), 1309-1311.

[11] MEIER, M.: Liouville theorems for nondiagonal elliptic system in arbitrary dimensions Math. Z. 176 (1981), 123-133.

[12] NečAS, J.: Introduction to the theory of nonlinear elliptic equations (Teubner-Texte zur Mathematik: Band 52). Leipzig: G. B. Teubner Verlagsges. 1983.

- [13] Neе̌As, J., и О:А. ОлЕЙник: Т'еремы Лиувилля для эллиптических систем. Докл. Акад. Наук СССР 252 (1980), 1312-1316. .

[14] NeČAs, J.; John, O., and J. STARÁ: Counterexample to the regularity of weak solution of elliptic systems. Comment. Math. Univ. Carolinae 21 (1980), 145-154.

[15] Stará, J., Daněček, J., and O. Johs: Liouville type condition and the interior regularity of quasilinear parabolic system (the case of BMO-solutions). Comment. Math. Univ. Carolinae 28 (1987), 103-109.

Manuskripteingang: 26. 09. 1988; in revidierter Fassung: 06. 12.1989

\section{VERFASSER :}

JosEF DANË̌EK

Katedra matematiky, fakulta stavební

Vysoké učení technické. Brno

Barvicova 85

CS -66237 Brno 